\title{
On the Search for a Level-Agnostic Modelling Language
}

\author{
Brian Henderson-Sellers ${ }^{1}$, Tony Clark ${ }^{2}$, and Cesar Gonzalez-Perez ${ }^{3}$ \\ ${ }^{1}$ Faculty of Engineering and Information Technology, University of Technology, \\ Sydney, PO Box 123, Broadway, NSW 2007, Australia \\ ${ }^{2}$ Middlesex University, The Burroughs, Hendon, London, NW4 4BT, UK \\ ${ }^{3}$ Institute of Heritage Sciences (Incipit) \\ Spanish National Research Council (CSIC) \\ Santiago de Compostela, Spain \\ brian.henderson-sellers@uts.edu.au, t.n.clark@mdx.ac.uk, \\ cesar.gonzalez-perez@incipit.csic.es
}

\begin{abstract}
The use of models is increasing in software engineering, especially within the MDE initiative. Models are usually communicated by visualizing them, typically using a graphical modelling language. The architecture commonly used to standardize a software engineering modelling language utilizes multiple levels despite the fact that the basic assumptions are only valid for a pair of levels. This has led several research groups to seek a means by which modelling languages can be created, and later standardized, without resorting to 'fixes' necessitated by the use of strict metamodelling and a multilevel hierarchy. Here, we describe a novel single-level approach based on 'everything is an object', which permits effective flattening of such a hierarchy, thus obviating all the paradoxical concerns in the literature over the last two decades.
\end{abstract}

Keywords: modelling, multi-level architecture, metamodelling, MDE.

\section{Introduction}

Contemporary and future engineering of information systems place an increasing emphasis on the use of models, either directly to aid design and implementation, in a more formal sense for code generation or as the backbone to model-driven engineering (MDE) (e.g. [1]) or model-based engineering [2]; although the costs and benefits of modelling for software development, and in particular the use of the Unified Modeling Language $^{\mathrm{TM}}\left(\mathrm{UML}^{\mathrm{TM}}\right)$, are not well evaluated (see e.g. [3]).

Models must be described in some way; typically using a notation (a.k.a. concrete syntax) associated with a modelling language. The language itself may be defined in many ways but typically a metamodel is used e.g. [4-6]. That metamodel must itself be defined, by a metametamodel. Together with the instances conformant to the model, this leads to an identification of four abstraction levels of interest to the modeller and metamodeller. Although in use for almost two decades, a four-layer architecture like that of the Object Management Group (OMG) raises some concerns both theoretically and 
pragmatically. This led several researchers (e.g. [7-9]) to seek a way of describing models and modelling languages without the use of this 'strict metamodelling' hierarchy of the OMG (see also [10-11] regarding the constraints of strict metamodelling). With a similar motivation is the architecture devised and utilized in the ISO/IEC 24744 standard [12] wherein the levels (known as 'domains') are aligned with practice rather than the theoretical notion of instanceOf used in strict metamodelling.

It is clear that there is no single approach to software metamodelling. Approaches based on the OMG strict metamodelling architecture have long been criticized, especially when applied to processes and methodologies (see summary in [13]) since the traditional strict metamodelling approach is unable to support enactment e.g. [14] i.e. it defines attributes at level M2, thus giving them values at M1 when what is actually needed is values at M0. This enactment support is provided by the architecture used by ISO/IEC 24744 [12] but at the expense of relying on powertype patterns (e.g. Fig. 1), which do not accord with the philosophy of strict metamodelling since they require a generalization relationship between consecutive levels. They also result in clabjects, like Dog in Fig. 1, where a clabject is an entity with both a class facet and an object facet [11].

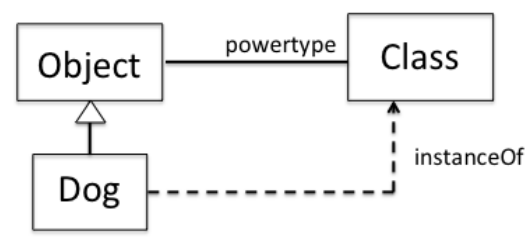

Fig. 1. Powertype pattern with an example clabject (Dog) that is conformant to it

In this paper, we ask the question: Is there a foundation that explains all the above? In particular, we seek a level-agnostic metamodelling style that will obviate the need for strict metamodelling and the contentious use of repeated instanceOf relationships between layers.

In Section 2, we describe the proposals to date for a level-agnostic approach, describing initial ideas that focussed just on a level agnostic notation, leading to a short discussion on the so-called ontological versus linguistic metamodel paradox and on the full-blown proposals for a level-agnostic language from Atkinson and colleagues published recently, which we will demonstrate partially fail in their aim. Section 3 introduces our proposal for a comprehensive approach to create a metamodelling infrastructure that is truly level-independent. Section 4 discusses some justification of our work, including some prototype tool implementation, whilst Section 5 concludes our presentation.

\section{Earlier Proposals}

Soon after the publication of the first version of the UML in 1997, concerns were raised about the notation used that was not consistent across models and metamodels. 
In Section 2.1 we outline the proposals made in 1999 and 2000 to improve on the UML's notation - ideas that were never adopted by the OMG. This led the same research team a few years later to investigate, in their modelling of processes, an idea called potency e.g. [15]. In using potency, an annotation is added to each attribute of the metamodel class indicating at which level 'below' it is necessary to allocate a value to the attribute - so-called deep instantiation.

\subsection{A Level-Independent Notation}

The first discussion on level-independent modelling languages focussed on the simpler concern of a level-independent notation. It was pointed out [11], [16] that underlining to indicate an object should also be applied to classes in the M1 model since they are also instances of the class Class in the M2 model. Coupling that with their suggestion that this necessitates the use of clabjects, particularly to represent M1-level entities, leads these authors to consider a notation that encompasses both the class facet of a clabject and its object facet: 'instance'-related features are indented with respect to 'type'-related features such that this convention applies at all Mx levels (of the OMG strict metamodelling architecture). Additionally, a superscript is optionally added after the name of the clabject to indicate the level.

\subsection{Ontological cf. Linguistic Metamodelling}

We describe here one example of a step in the search for a comprehensive theory of metamodelling. Atkinson and Kühne (2003) argue that there is a second multi-level hierarchy, based on ontological classification (left hand side of Fig. 2 labelled Ox $(\mathrm{x}=1$ to 3$))$. This argument was later developed into the Orthogonal Classification Architecture [17] - as shown in Fig. 3. The first question is how this does or does not fit with the OMG's four-level architecture. If we consider a model such as that depicted in the left hand side of Fig. 2 (i.e. Rover-Collie-Breed), it might be argued that there is a correspondence between these three 'levels' (connected by two instanceOf relationships) and the lower three levels (M0, M1 and M2) of the OMG architecture. In other words, if an object (M0) is an instance of a class (M1), which, in turn, is an instance of a class in the metamodel (M2), then Breed in Fig. 2 should be equivalent to a metaclass. This is, however, problematical since clearly Breed is of a very different nature to classes in the M2 metamodel that defines OMG's Unified Modeling Language. Furthermore, the double instanceOf is not permitted from a language use perspective e.g. [18], [19]. Consequently, in attempting to rationalize this different nature, Atkinson and colleagues [17] proposed the Orthogonal Classification Architecture (OCA) as shown in Fig. 3. Here, linguistic classification is depicted on the vertical axis and ontological classification on the horizontal. Indeed, we concur that there are indeed two orthogonal issues here - but only one (the links between models, ontologies, metamodels etc. as discussed in [6]) has relevance to the broader search for a 'better' approach to software modelling language engineering. So-called ontological metamodelling has as its focus 'modelling' and nothing else. 


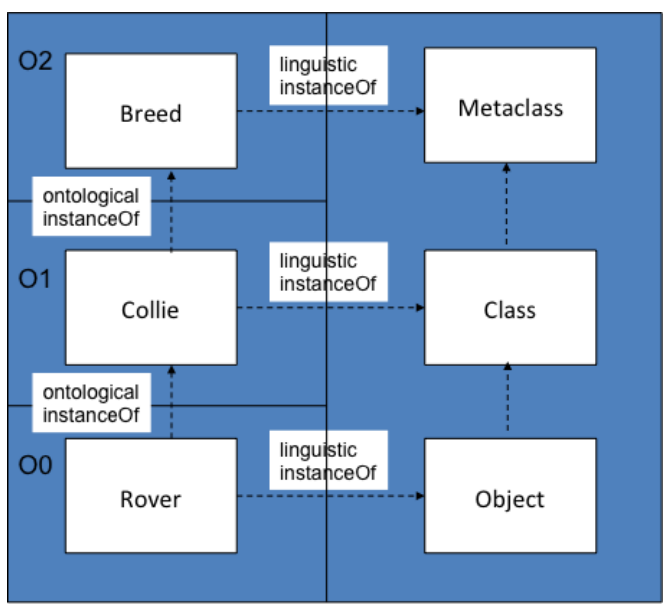

Fig. 2. Linguistic versus ontological metamodelling (modified from [6])

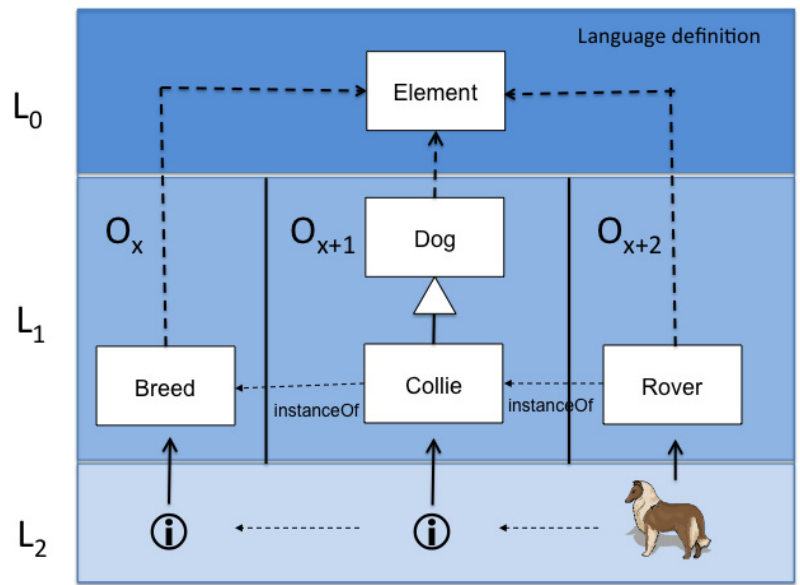

Fig. 3. Orthogonal classification architecture using the example of Fig. 2 elaborated as in [20]. Note that in [20] relabelling of both Ox and Lx have a reversed incremental direction cf. Fig. 2.

It is, however, worth noting that the resultant architecture (in Fig. 3) has many similarities with the more recently derived from language use considerations as shown here in Fig. 4 (where, importantly, Rover is correctly shown as an instance of Dog not an instance of Collie as in Fig. 3). In both figures, we see a 'metamodel layer' $\left(\mathrm{L}_{0}\right.$ in Fig. 3), a combined objects+class modelling layer $\left(\mathrm{L}_{1}\right)$ and a correspondence to real objects $\left(\mathrm{L}_{2}\right)$ - although in the $\mathrm{L}_{2}$ layer of Fig. 3 , there is a mixture of objects and conceptual representations (see speech act discussion in [21] and philosophical discussion in [22] for alternative viewpoints). 


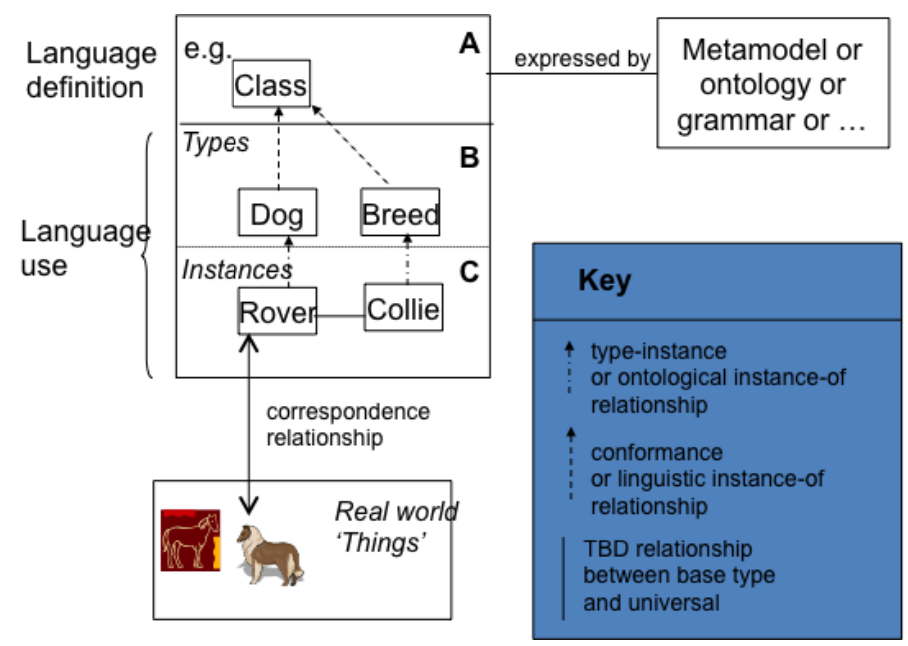

Fig. 4. Complete multilevel framework based on language use - to replace the OMG's strict metamodelling architecture when modelling in information systems development and software engineering (after [6])

\subsection{Pan-Level Model (PLM) and Level-Agnostic Modelling Language (LML)}

Having proposed the OCA (Fig. 3), Atkinson and colleagues [20] then introduced an infrastructure to support it, consisting of a Pan-Level Metamodel (PLM), together with a Root Ontological Model (ROM), a multilevel constraint language and a multilevel visualization language. Of particular interest here is the PLM, which is depicted as a metamodel containing a number of 'metalevel' elements, some of which are in fact clabjects (PNode and PRelationship). To support multiple ontological levels, it is necessary for the PLM to support both generalization (PGeneralization) and instantiation (PInstantiation). All the instantiable types in the PLM have a potency property. However, when used later, the PLM is said not to be equivalent to OMG's M2 level but to the M3 level i.e. "it plays the same role as the MOF ... in the OMG ... modelling infrastructure" [7]. [There are other technical issues with the PLM such as its heterogeneous treatment of fields and methods with regard to nodes and relationships.]

Atkinson et al. [7] then seek to extend the PLM into a fully-fledged modelling language: the LML or Level-agnostic Modelling Language, which also includes a concrete syntax (notation), the abstract syntax being supplied by the PLM (Fig. 5).

The LML thus appears to be targeted at offering alternative modes of representation for the $\mathrm{O}_{2}-\mathrm{O}_{1}-\mathrm{O}_{0}$ chain and particularly at modelling products and product types e.g. [23,24]. In other words, it is aimed not at creating a mono-level language in toto but primarily at documenting models, however complex, that reside within a single linguistic layer (labelled $\mathrm{L}_{1}$ in Fig. 5). This, these authors claim, substantiates the appellation of 'ontological'. However, in doing so they eschew all the trappings of an ontological (meta)model such as the UFO (Unified Foundational Ontology) [25,26]. A true 'ontological' model, still totally within $\mathrm{L}_{1}$, would be one that utilized the ideas of sortals, universals etc. e.g. [27, 28, 6]. 


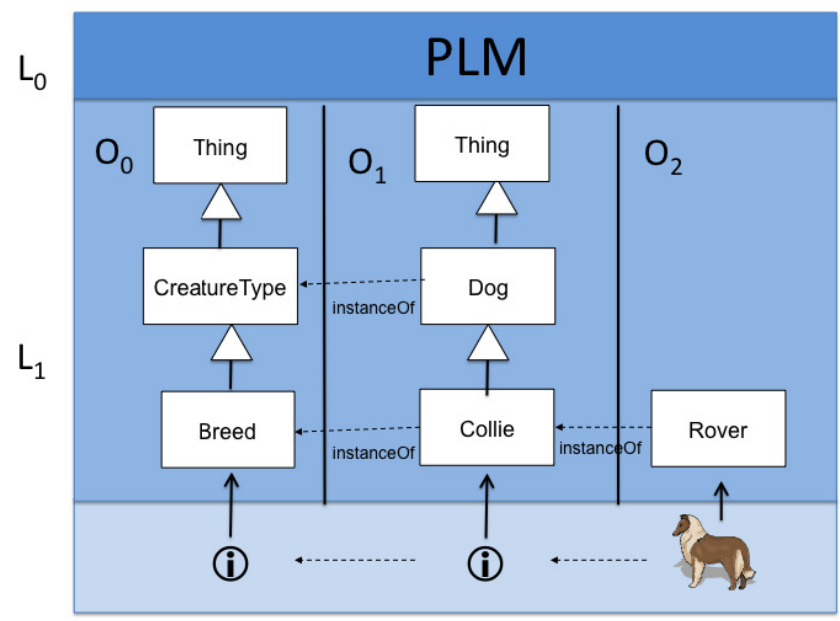

Fig. 5. Extension made in [7] to Fig. 3 towards a level-agnostic modelling language (LML)

However, LML-utilizing diagrams like Fig. 5 still focus on the Rover-Collie-Breed chain of, arguably, three different ontological levels. That means there is still the problematical double instanceOf problem exemplified earlier (Fig. 2) (see also [21]). Thus, whilst we agree that there are indeed two orthogonal issues (as above), the one identified (e.g. [29]) as 'ontological modelling' does NOT use the truly ontological solution but rather relies on linguistic elements, in particular the introduction of a Clabject class into the language-defining metamodel for LML [7]. Such an introduction (of Clabject) into the metamodel does legitimize the existence of individual clabjects in the $\mathrm{L}_{1}$ layer. However, it is unclear how to represent such instantiation using set theory (cf. [6]) and, furthermore, it disguises the meaning of clabject instantiation by imposing a potency overlay on to each clabject - thus making it appear compatible with the strict metamodelling architecture of the OMG. In other words, clabject instantiation, although not clearly defined by these authors, must be understood to be some combination of regular (linguistic) instantiation and generalization in order to rationalize the fact that some attributes are instantiated without value assignment and some with value assignment (see discussion in [21]).

There is one other concern that has not yet been explained satisfactorily. The idea of potency [30] was created to support process modelling i.e. multilevel linguistic modelling in the terminology used in this section. What is therefore confusing is its use in ontological (meta)modelling. It is crucial to the arguments above that potency is used to permit attributes of, say, Breed to be made available to Rover, two ontological layers lower. The problem is that if the relevant metaclass in the PLM has an attribute $\mathrm{Y}$ with potency $\mathrm{m}$, then Thing in $\mathrm{O}_{0}$ in Fig. 5 has a potency value of $m-1$ for $\mathrm{Y}$ and thus Breed's Y attribute similarly has a potency value of $m$ - 1 . When Breed is instantiated to Collie, this potency value becomes $m$ - 2 . Now, Thing also exists in $\mathrm{O}_{1}$ and its $\mathrm{Y}$ attribute also has a potency value of $m$ - 1 . Since Collie is a direct descendant of Thing, it too has a potency value of its $\mathrm{Y}$ attribute of $m-1$. But we have just shown that Collie must have a potency value of $m-2$. Hence, we have a contradiction with this (new) use of potency. This problem needs resolution before this approach can be 
accepted as useful in multi-level metamodelling (taking linguistic and ontological modelling as a single package).

\section{A New Modelling Approach in Which 'Everything Is an Object'}

In this section, we introduce our initial assumption that a better (than strict metamodelling) approach to metamodelling and software language engineering is to start with the idea that 'everything is an object'. [Of course, not everything is an object because an object, in software terms, must either refer to a substantial individual (brute fact), a universal (a concept) or an institutional fact [31].]

Some of the underlying rationale for proposing this starting point is that in philosophy and language use, the referent of the subject of a speech act (typically a sentence) is a particular. A particular has individuality. However, this may be an instance with substance e.g. a single human being or it may be a universal - for instance, the concept of person regarded as a single thing; i.e., there are many things, both singular and collections, that can be thought of as 'an object'.

In addition, software language engineering supports the universal powertype pattern of Class-Object [32] i.e. the Class class is a powertype of the Object class. Using this (M2-level) pattern, we see that a user-defined (M1) class (actually a clabject) such as Dog is an instance of Class and a subtype of Object (Fig. 1).

Consequently, we claim that this approach is both necessary and sufficient to support all the current proposals for foundations of meta-modelling. Once accepted, this leads to a 'grand unification', making, inter alia, the creation of a compatible suite of integrated software support tools significantly easier. For example, a unified representation makes it possible to generate a single model editor, or a single transformation engine, that works over any M-level, whilst leaving open the incremental definition of ever more specialised editors or transformation engines that support specific languages.

\subsection{MML as a Basic Infrastructure}

Álvarez et al. [33] propose a modelling language named MML (Meta-Modeling Language), which uses package specialization based on that in Catalysis [34]. The models.concepts package describes valid metamodels whilst the instances.concepts package describes valid instances (e.g. UML models). Package contents are Class and Attribute; and Object and Slot respectively (Fig. 6). There is also a third package linking these elements. MML is thus both a metamodelling language and a kernel language such that a language such as UML could be called both a specialization and an instance of the MML metamodel [33].

By partitioning the fundamental components of a language definition as model (classes) versus instance and concepts (logical elements of a language such as class or object) versus syntax (textual or graphical representation), Álvarez et al. [33] create an architecture that has only classifiers (at the metalevel) and instances (at all other metalevels). In this conceptualization, it is argued that everything is an object (instance) in that, for instance, "a class can always be thought of as an instance of the 


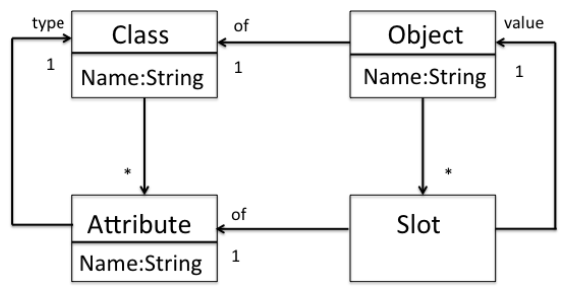

Fig. 6. Contents of the three packages of MML (as recommended in [33])

metaclass Class". Thus there are two representations of the same entity, related by a one-to-one mapping, which they call the ' $G$ mapping' - a meta-instantiation. [This has similar results to OOLang of [35], as shown in their figure 26.] Furthermore, to stress that this approach is not strictly strict metamodelling, they label their layers as $\mathrm{M}_{\mathrm{n}}{ }$ where $\mathrm{M}^{\prime}{ }_{0}$ is the top-most layer. Then, for a model $\mathrm{X}$ at layer $\mathrm{M}_{\mathrm{n}}{ }_{\mathrm{n}}$ we can create a 'lower level' model as an instantiation:

$$
X\left(M^{\prime}{ }_{n}\right) \cdot G=X\left(M^{\prime}{ }_{n+1}\right)
$$

Even lower levels are then created simply by an additional meta-instantiation or Gmapping:

$$
\mathrm{X}\left(\mathrm{M}^{\prime}{ }_{\mathrm{n}}\right) \cdot \mathrm{G} \cdot \mathrm{G}=\mathrm{X}\left(\mathrm{M}^{\prime}{ }_{\mathrm{n}+2}\right)
$$

It is worth noting that the ' $\mathrm{G}$ mapping' is an approach that is not dissimilar from that proposed in Fig. 7 i.e. a clabject, although not so named by these authors; rather they describe it as an implicit G-mapping since classes can only exist at the $\mathbf{M}_{0}{ }_{0}$ level everything else must be an object. They note that this approach is not restricted to a small number of layers, although only this small number has any practical application.
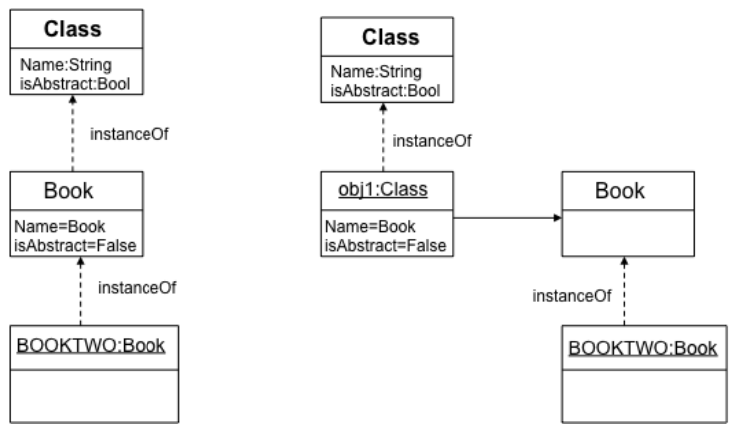

Fig. 7. On the left-hand side, Book is shown as a class that is also an instance of Class, which is impossible within the conventional object-oriented paradigm. On the right-hand side, Book is shown as a class that is specified by the object obj1 through a forward-looking isotypical interpretive mapping (the solid arrow) (modified slightly from [35]). 

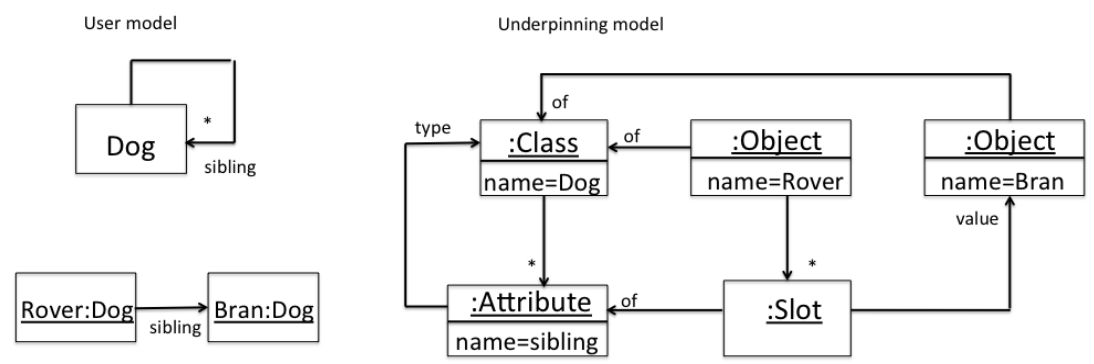

Fig. 8. Example of a user model (regular class diagram representation) and its underpinning model using MML

To model these ideas, Álvarez et al. [33] note that for an element in a given level (e.g. Class at level $\mathbf{M}_{0}^{\prime}$ ), an operation corresponding to the G-mapping is added. This mapping creates an Object at level $\mathrm{M}_{1}{ }_{1}$ with a slot 'of=Class'. Thus, we can depict a 'normal' class diagram (left hand side of Fig. 8) and its underlying model (right hand side). Whilst, in essence, Fig. 8 is a single-level model (see below), these authors [33] feel the necessity to 'carve it up' into a number of non-linear 'layers', as shown in Fig. 9. This has a striking similarity to Fig. 4 in that there is (a) language definition metamodel in Fig. 9; and (b) language use that refers to both types and instances user model plus user objects in Fig. 9.

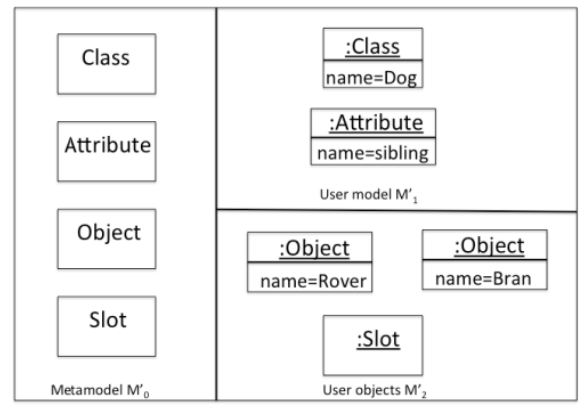

Fig. 9. Elements of the model of Fig. 8 positioned in three non-linear 'levels': Metamodel, User model and User objects (modified from [33])

We are not proposing to define a basis for metamodelling in terms of a logic. Such an approach would require higher-order features as discussed in [36, 37] with their associated complexity and paradoxes. We propose to use a constructive approach in the sense of the MML calculus described in [38] where a small collection of primitive, externally defined rules govern a language for constructing and processing objects. In this way, self-reference is constructed by infinite unfolding, rather than constrained to exist as with a logic, thereby avoiding paradoxes that beset higher-order reasoning. In such a calculus, operational rules will define 'type' compliance and reflexivity is achieved by requiring a system to comply with itself. In characterizing metamodelling using this approach we must be careful to acknowledge that there are a family of calculi that vary in terms of the primitive constructs used to achieve reflexivity. 
The benefits of taking this approach arise because of the unification of core representation for all elements. Consider developing an editor for a range of languages. Such a tool would be useful for instances of a language or for developing the language itself. If everything is ultimately an object then the editor written against the definition of an object can be supplied with anything: from a (meta-)*model to ground instances. If the base language uses patterns to detect models, then features of the base editor can be extended to provide support for type-level editing. Detecting increasingly complex configurations of objects can support domain-specific languages. Furthermore, our approach naturally leads to the situation where the editor is applicable to itself.

Achieving a unification of representation and reflexivity is an advance on other approaches where tools (e.g. editors) must be repeated for different type-levels and where the semantics of each new language must be defined externally on a case-bycase basis instead of being based on a small set of unifying rules.

\subsection{Extending the Basic Infrastructure: A New Proposal}

Applying and extending these ideas, we can create a modelling architecture in which 'everything is an object'. We commence with a single entity (represented in Fig. 10 by a class), named Object. Any object is of a specific class, so we now add a second class, called Class, such that an object is 'of' a class, following [33]. Objects must also have slot values (and hence classes have named attributes) - thus recreating the model in Fig. 6.

Now, since everything is an object, we must also have an inheritance relationship between Class and Object and a recursive relationship on Class to allow for classclass generalization hierarchies (Fig. 10). Constraints must also be enforced to prevent repeated inheritance and other standard constraints on generalization hierarchies (for further details see [39]).

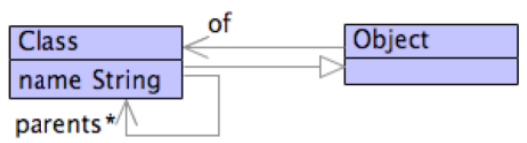

Fig. 10. Class and Object in the new 'everything is an object' modelling infrastructure

It is crucial to appreciate that the consequence of Fig. 10 is that tools and methods can be defined against the universal object format that consequently apply to instances, models, metamodels and metametamodels without further modification. It is also important to note that, since everything is an object, we can take a set-based approach whereby Object is associated with a universal set of objects and specialization denotes the sub-set relation.

Fig. 11 elaborates Fig. 10 with the key features of the approach that achieve the necessary meta-circularity. The combination of objects and slots provides the universal representation. The rest of Fig. 11 provides the specializations of Object necessary to define Class in terms of its structure (Attribute) and the constraints that apply to its instances (Constraint and Function). The classes SeqType and Sequence are necessary 
to provide types (and their instances) that are used in attributes with multiplicity *. Packages are collections of classes that are used to define languages. We make a distinction between information (expressed fundamentally in terms of objects) and the presentation of information shown in terms of a diagram (or any other concrete syntax). It is important to appreciate that we are always dealing with collections of objects and slots, although we may choose to draw these in a more convenient form.

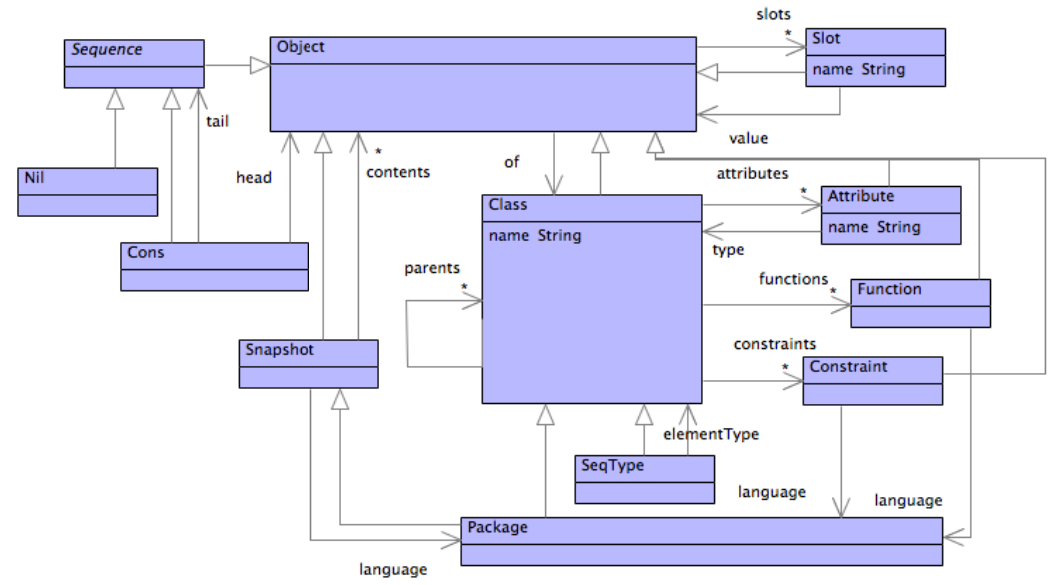

Fig. 11. Elaboration of Fig. 10, depicted as a (UML) class diagram

\section{Discussion}

The approach described in the previous section and outlined in Fig. 11 aims to provide a level-agnostic meta-circular basis for language-based modelling. The kernel of the approach provides a universal format for models at any level and supports levelagnostic tooling. The kernel has been used as the basis of a commercial tool called XMF-Mosaic (provided as an open source platform at https://bitbucket.org/ xmodeler/xmodrel) [40] that has been used as a basis for tooling with customers in diverse fields including finance, where it was is to implement a language for Enterprise Architecture for a large multi-national bank, aerospace, where it is used to implement a domain-specific language and code generator for a product-line of aircraft communication infrastructure, and telecoms, where it is used to automatically generate code conforming to a network standard (see [40, 41]). The XMF kernel (roughly 2 KLOC, based on approaches such as Smalltalk and ObjVLisp [42]) is based on the approach proposed in this paper and the rest of the system (roughly 200 KLOC) including language processors, model-management tools, and graphics editors is defined in terms of the kernel and achieves complete user-level transparency and extensibility, and subsumes approaches such as clabjects and powertypes. The Kernel includes behavioural features that are based on extension of the class Function defined in Fig. 11. The entire toolset is written in the Kernel using language definitions supported by the provided language engineering features (again written in the 
Kernel). The entire system runs on a small virtual machine written in Java that provides access to external libraries such as IO and graphics. Without an object-based, reflexive approach this would not have been possible. XMF-Mosaic was reviewed in [43] as the leading tool for supporting abstraction in software engineering.

Although not shown in Fig 11, metaclasses are those distinguished classes that directly or indirectly inherit from the class Class and thereby inherit the facility to give their instances (classes) the ability to specify constraints that, in turn, govern their instances. This three-level relationship has been referred to as a Golden Braid [44] and can be replicated through inheritance as many times as is required. Metaclasses (and their containment through Package) are the basis for language definition since packages can be associated with Snapshots whose contents can (due to the universal representation) be instances, models, metamodels etc. The language-based kernel relies on semantics expressed as constraints and functions. The semantics of these languages are necessarily external to the kernel, although they can be made extensible through features offered by the kernel using a mechanism called a meta-object protocol (MOP). This will complete meta-circularity and will be explored in more detail in [39].

The proposed language-based approach allows new modelling languages to be defined as modular units; we claim that this subsumes other approaches to metamodelling by allowing them to be defined and used as languages. For example, a potency-based language [7-9] can be defined as a package that contains an extension to Attribute called PotentAttribute equipped with an extra numerical attribute indicating the level of instantiation that must hold (by traversing 'of' links) before a slot occurs in instances of a new type of class called PotentClass. The approach also supports clabjects [11] without further elaboration since everything is an object and therefore level-agnostic. Level-n information can be freely mixed with level-m (where $m<>n$ ) information and furthermore the patterns that define such mixtures can be precisely expressed as languages, providing a basis for a collection of formalized clabject-like languages. The standard class diagram presentation for such languages would clearly be inadequate since there would be no way to present instance-level information, although our approach associates one or more presentation mappings for each language and therefore presentations for clabject-based languages could use indentation as described in Section 2.1.

Finally, a language with powertypes (e.g. [12]) can be supported by defining a new type of class called PowerClass that contains an extra attribute called 'type' whose type is Class. This results in models written in the new language containing classes with links to other classes in the model; for example, Rover may be an instance of Dog where dog is a PowerClass with a type link to the Breed class.

The potential disadvantage is the apparent conflict with standard ontological representations that utilize a four-category ontology e.g. [45, 46]. In this approach, 'things' are either entity types (a.k.a. universals) or individuals (a.k.a. particulars). Each of these may have a characteristic of being an endurant or a perdurant. An alternative ontological viewpoint is presented in [47] based on tropes, which are instances of properties (i.e. particulars) that are existentially dependent upon other particulars. In both philosophies (universalism and trope theory [47]), it would appear that our 'everything is an object' could possibly be equated with 'everything is a thing' or 
'everything can be particularized' [48] such that Object in Fig. 11 would be regarded as an abstract super-category of ontological Substantial Universals and Moment Universals (both subtypes of Endurant Universal: cf. figure 8-9 of [25]) as well as their counterpart subtypes of Endurant Particular (e.g. figure 3 of [26]). This further linking of language engineering and ontological engineering must, however, remain the topic of additional, detailed research on foundational ontology level modelling (see also [21]). A remaining challenge is to investigate whether this proposal avoids wellknown mathematical and linguistic paradoxes e.g. Russell and Grelling-Nelson.

\section{$5 \quad$ Conclusions and Future Work}

The past couple of decades have seen the growth of the use of models at all stages of information systems development and software engineering, especially within the MDE initiative. Models are typically communicated by visualizing them, typically using a graphically based modelling language. The architecture commonly used to standardize a software engineering modelling language utilizes multiple levels despite the fact that the basic assumptions are only valid for a pair of levels. This has led several research groups to seek a means by which modelling languages can be created, and later standardized, without resorting to 'fixes' necessitated by the use of strict metamodelling and a multilevel hierarchy.

In this paper, we have first outlined some of these fixes (described in detail in [21]), particularly in terms of the so-called linguistic versus ontological paradox that led one group of researchers to the development of a Pan Level Model (PLM) and a Levelagnostic Modelling Language (LML). Notwithstanding their claims that this approach is level-agnostic, we note (e.g. Fig. 5) that it still requires two levels, although all modelling is now done within a consistent framework (labeled $\mathrm{L}_{1}$ in Fig. 5).

Here, we have outlined the basis for a novel approach that it truly single- level. This is based on the notion that 'everything is an object'. The creation of a single level effectively flattens the previous research and practical results across any multilevel hierarchy currently in use and thus not only obviates the paradoxical concerns in the literature (as discussed here in Section 2) but also offers an intriguing and simplified future for tool builders and modelling language users. In addition, we have shown that this approach provides an integrated base that can be tailored to support languages using potency, clabjects and/or powertypes.

The core elements, shown in Figs. 10 and 11, are the core for future extensions that will support role modelling as well as object behaviour using operations together with a mechanism to be based on snapshots [39].

\section{References}

1. Sánchez-Cuadrado, J., de Lara, J., Guerra, E.: Bottom-up meta-modelling: An interactive approach. In: France, R.B., Kazmeier, J., Breu, R., Atkinson, C. (eds.) MODELS 2012. LNCS, vol. 7590, pp. 3-19. Springer, Heidelberg (2012) 
2. Selic, B.: What will it take? A view on adoption of model-based methods in practice. Software and Systems Modeling 11, 513-526 (2012)

3. Chaudron, M.R.V., Heijstek, W., Nugroho, A.: How effective is UML modeling? Softw. Sys. Model. 11, 571-580 (2012)

4. Kühne, T.: Matters of (meta-)modeling. Softw. Syst. Model. 5, 369-385 (2006)

5. Henderson-Sellers, B.: Bridging metamodels and ontologies in software engineering. J. Systems Software 84, 301-313 (2011)

6. Henderson-Sellers, B.: On the Mathematics of Modelling, Metamodelling, Ontologies and Modelling Languages, Springer Briefs in Computer Science. Springer (2012)

7. Atkinson, C., Kennel, B., Goß, B.: The level-agnostic modeling language. In: Malloy, B., Staab, S., van den Brand, M. (eds.) SLE 2010. LNCS, vol. 6563, pp. 266-275. Springer, Heidelberg (2011)

8. Atkinson, C., Kennel, B., Goß, B.: Supporting constructive and exploratory modes of modeling in multi-level ontologies. In: Procs. 7th Int. Workshop on Semantic Web Enabled Software Engineering, Bonn (October 24, 2011)

9. Kennel, B.: A unified framework for multi-level modeling. PhD Thesis, Univ. Mannheim, Germany, 229 p. (2012)

10. Atkinson, C.: Metamodelling for distributed object environments. In: Procs. First International Enterprise Distributed Object Computing Workshop (EDOC 1997), pp. 90-101. IEEE Computer Society, Brisbane (1997)

11. Atkinson, C.: Supporting and applying the UML conceptual framework. In: Bézivin, J., Muller, P.-A. (eds.) UML 1998. LNCS, vol. 1618, pp. 21-36. Springer, Heidelberg (1999)

12. ISO/IEC: Software Engineering - Metamodel for Development Methodologies, ISO/IEC 24744. International Organization for Standardization, Geneva (2007)

13. Gonzalez-Perez, C., Henderson-Sellers, B.: Metamodelling for Software Engineering, p. 210. J. Wiley \& Sons, Chichester (2008)

14. Aharoni, A., Reinhartz-Berger, I.: A domain engineering approach for situational method engineering. In: Li, Q., Spaccapietra, S., Yu, E., Olivé, A. (eds.) ER 2008. LNCS, vol. 5231, pp. 455-468. Springer, Heidelberg (2008)

15. Atkinson, C., Kühne, T.: The essence of multilevel metamodeling. In: Gogolla, M., Kobryn, C. (eds.) UML 2001. LNCS, vol. 2185, pp. 19-33. Springer, Heidelberg (2001)

16. Atkinson, C., Kühne, T.: Meta-level independent modelling, In: Procs. In: Procs. Int. Workshop on Model Engineering (Part of ECOOP 2000), Sophia Antipolis, Cannes, France (2000)

17. Atkinson, C., Kühne, T.: Concepts for comparing modeling tool architectures. In: Briand, L.C., Williams, C. (eds.) MoDELS 2005. LNCS, vol. 3713, pp. 398-413. Springer, Heidelberg (2005)

18. Searle, J.R.: Speech Acts. An Essay in the Philosophy of Language. Cambridge Univ. Press, p. 203 (1969)

19. Eriksson, O.: Pers. Comm (2012)

20. Atkinson, C., Gutheil, M., Kennel, B.: A flexible infrastructure for multilevel language engineering. IEEE Trans. Software Eng. 35(6), 742-755 (2009)

21. Henderson-Sellers, B., Gonzalez-Perez, C., Eriksson, O., Ågerfalk, P.: Ptolemaic metamodelling? The need for a paradigm shift. In: Cueva Lovelle, J.M., Pelayo García-Bustelo, C., Sanjuán Martínez, O. (eds.) Progressions and Innovations in Model-Driven Software Engineering. IGI Global (in press, 2013)

22. Henderson-Sellers, B., Gonzalez-Perez, C., Walkerden, G.: An application of philosophy in software modelling and future information systems development. In: Franch, X., Soffer, P. (eds.) CAiSE 2013 Workshops. LNBIP, vol. 148, pp. 329-340. Springer, Heidelberg (2013) 
23. Kühne, T.: Making modeling languages fit for model-driven development. paper presented at ATEM 2007 (Part of MoDELS 2007). IEEE online publication (2007)

24. Atkinson, C., Kühne, T.: Reducing accidental complexity in domain models. Software and Systems Modeling 7(3), 345-359 (2008)

25. Guizzardi, G.: Ontological foundations for structural conceptual models. CTIT PhD Thesis Series, No. 05-74, Enschede, The Netherlands (2005)

26. Guizzardi, G., Wagner, G.: Towards ontological foundations for agent modelling concepts using the unified fundational ontology (UFO). In: Bresciani, P., Giorgini, P., HendersonSellers, B., Low, G., Winikoff, M. (eds.) AOIS 2004. LNCS (LNAI), vol. 3508, pp. 110124. Springer, Heidelberg (2005)

27. Guizzardi, G., Ferreira Pires, L., van Sinderen, M.: An ontology-based approach for evaluating the domain appropriateness and comprehensibility appropriateness of modeling languages. In: Briand, L.C., Williams, C. (eds.) MoDELS 2005. LNCS, vol. 3713, pp. 691-705. Springer, Heidelberg (2005)

28. Guizzardi, G., Wagner, G., Guarino, N., van Sinderen, M.: An ontologically well-founded profile for UML conceptual models. In: Persson, A., Stirna, J. (eds.) CAiSE 2004. LNCS, vol. 3084, pp. 112-126. Springer, Heidelberg (2004)

29. Atkinson, C., Kühne, T.: Model-driven development: a metamodelling foundation. IEEE Software 20, 36-41 (2003)

30. Atkinson, C., Kühne, T.: Processes and products in a multi-level metamodelling architecture. Int. J. Software Eng. and Knowledge Eng. 11(6), 761-783 (2001)

31. Eriksson, O., Ågerfalk, P.J.: Rethinking the meaning of identifiers in information infrastructures. J. AIS 11(8), 433-454 (2010)

32. Johnson, R., Woolf, B.: The type object pattern. In: Pattern Languages of Program Design, vol. 3, pp. 47-65. Addison-Wesley (1997)

33. Álvarez, J., Evans, A., Sammut, P.: Mapping between levels in the metamodel architecture. In: Gogolla, M., Kobryn, C. (eds.) UML 2001. LNCS, vol. 2185, pp. 34-46. Springer, Heidelberg (2001)

34. D'Souza, D., Wills, A.C.: Objects, Components and Frameworks with UML: The Catalysis Approach. Addison-Wesley (1998)

35. Gonzalez-Perez, C., Henderson-Sellers, B.: Modelling software development methodologies: a conceptual foundation. J. Sys. Softw. 80(11), 1778-1796 (2007)

36. Halpin, T.: Higher-order types and information modeling. In: Siau, K. (ed.) Advanced Topics in Database Research 4, pp. 218-237. Idea Publishing Group, Hershey (2005)

37. Halpin, T., Morgan, T.: Information Modeling and Relational Databases, 2nd edn. Morgan Kaufmann (2008)

38. Clark, T., Evans, A., Caskurlu, B.: The meta-modeling language calculus: Foundation semantics for UML. In: Hussmann, H. (ed.) FASE 2001. LNCS, vol. 2029, pp. 17-31. Springer, Heidelberg (2001)

39. Clark, T., Henderson-Sellers, B., Gonzalez-Perez, C.: Formalizing a new level-agnostic modelling language, paper in preparation (2013)

40. Clark, T., Willans, J.: Software Language Engineering with XMF and XModeler. In: Mernik, M. (ed.) Formal and Practical Aspects of Domain-Specific Languages: Recent Developments. IGI Global (2013)

41. Achilleos, A., Georgalas, N., Yang, K.: An open source domain-specific tools framework to support model driven development of OSS. In: Akehurst, D.H., Vogel, R., Paige, R.F. (eds.) ECMDA-FA 2007. LNCS, vol. 4530, pp. 1-16. Springer, Heidelberg (2007)

42. Cointe, P.: Metaclasses are first class: the ObjVlisp model. ACM SIGPLAN Notices 22(12), 156-162 (1987) 
43. Helsen, S., Ryman, A., Spinellis, D.: Where's my Jetpack? IEEE Software 25(5), 18-21 (2008)

44. Hofstadter, D., Bach, G.E.: An Eternal Golden Braid. Basic Books (1979)

45. Lowe, E.J.: The Four-Category Ontology: A Metaphysical Foundation for Natural Science. Oxford Univ. Press (2006)

46. Laarman, A., Kurtev, I.: Ontological metamodeling with explicit instantiation. In: van den Brand, M., Gašević, D., Gray, J. (eds.) SLE 2009. LNCS, vol. 5969, pp. 174-183. Springer, Heidelberg (2010)

47. Guizzardi, G., Masolo, C., Borgo, S.: In defense of a trope-based ontology for conceptual modeling: An example with the foundations of attributes, weak entities and datatypes. In: Embley, D.W., Olivé, A., Ram, S. (eds.) ER 2006. LNCS, vol. 4215, pp. 112-125. Springer, Heidelberg (2006)

48. A suggestion of an anonymous reviewer (2013) 